\title{
Inspection of diesel engine injectors by several electromagnetic nondestructive methods
}

\author{
G. Vértesy ${ }^{\mathrm{a}, *}$, P. Balassa ${ }^{\mathrm{b}}$, A. Gasparics ${ }^{\mathrm{a}}$, I. Tomášc ${ }^{\mathrm{c}}$ and I. Mészáros ${ }^{\mathrm{b}}$ \\ ${ }^{a}$ Centre for Energy Research, Institute of Technical Physics and Materials Science, H-1121 Budapest, \\ Hungary \\ ${ }^{\mathrm{b}}$ Department of Materials Science and Engineering, Budapest University of Technology and \\ Economics, H-1111 Budapest, Hungary \\ ${ }^{\mathrm{c}}$ Institute of Physics ASCR, Prague 18221, Czech Republic
}

\begin{abstract}
Improved eddy current techniques and a method of magnetic hysteresis measurement were applied for nondestructive inspection of thickness of the hardened surface layer of the steel injectors of diesel engines. A monotonic correlation was found with enough sensitivity and acceptable signal/noise ratio between the electromagnetic parameters and the thickness of the layer. Both the hysteresis and the eddy current methods show very similar types of the correlation. Based on these results, practical application of these novel methods can be realized instead of the presently used destructive tests for inspection of the steel injectors of diesel engines.
\end{abstract}

Keywords: Electromagnetic nondestructive testing, diesel engine injector, eddy current testing, magnetic hysteresis measurements, magnetic Adaptive Testing

\section{Introduction}

Nondestructive testing (NDT) methods for inspection of different parts of machineries are widely used in many areas. Among them electromagnetic methods are rather well developed, have a lot of advantages compared with other techniques and they play a dominant role in everyday operation of power plants, pipeline networks, car industry, etc.

Surface hardening is commonly applied to improve mechanical properties of industrial components by achieving increased strength and toughness at the surface while still retaining ductility at the core. This offers advantages over no heat treatment by increasing service life of a component, and over through hardening by reducing heat treatment cycle time and cost. From practical point of view, determining the depth of hardened layer is important in quality control process which is traditionally performed by the time-consuming and costly destructive method of micro-hardness testing. The use of nondestructive evaluation (NDE) techniques for measurements of case depth in surface hardened components has

\footnotetext{
${ }^{*}$ Corresponding author: G. Vértesy, Centre for Energy Research, Institute of Technical Physics and Materials Science, H1121 Budapest, Konkoly Thege Miklós út 29-33, Hungary. Tel.: +36 1 3922677; Fax: +36 1 3922226; E-mail: vertesy.gabor @energia.mta.hu.
} 
increased in importance for quality control of both new and remanufactured industrial components. Increased quality control via NDE leads to improved safety, which in turn results in fewer warranty claims and thus higher profits.

Magnetic measurements, such as magnetic hysteresis and Barkhausen effect (BE) signal [1,2], have the potential to serve as effective methods for determining case depth in surface-hardened steel [3-9] since both techniques are sensitive to microstructure.

Eddy current test is another non-destructive technique which can be performed rapidly for this purpose (see e.g. [10]). Since the electromagnetic responses are sensitive to chemical composition and the microstructure of the materials under investigation, it can be used to determine the depth of the hardened layer in steels and cast iron parts due to the difference in magnetic properties of the hardened layer in comparison with the core of the specimens. There are numerous advantages of this technique e.g. high sensitivity; rapid scanning, contact-less inspection, and its versatility contribute to its widespread utilizations. This technique can also be utilized for the evaluation of metallurgical variations in addition to defect detection [11]. For example, Konoplyuk could establish an appropriate relation between the hardness of ductile cast iron and the primary and secondary voltages of eddy current signals [12]. Uchimoto [13] found the same relation for gray cast iron and they could determine mechanical properties of cast ductile iron such as elongation and tensile strength using nondestructive eddy current method. Relationship between the case depths of induction hardened cast iron parts and eddy current outputs (primary and secondary voltages, as well as normalized impedance) was studied in [14].

The purpose of the present work is the inspection of surface hardening of diesel engine injectors. These steel injectors are important parts of engines. In car industry there are very high requirements, according to ISO TS. Because of this the components of engines should be very rigorously tested before use. A part of the manufacturing process of injectors is surface heat treatment. During or after this process thickness of the trained surface layer should be checked. Industrially it is presently done by the so called TCD (Total Case Depth) technique, which is destructive, complicated and the accuracy is not very satisfactory.

We studied test parts of injectors by two ways of eddy current testing and by a way of magnetic hysteresis measurement. In spite of the fact that surface hardening can be controlled by different NDT methods (see above) to our best knowledge, nondestructive electromagnetic methods have not been applied for the inspection of industrial diesel engine injectors till now, the majority of reported investigations were performed on laboratory samples.

Another novelty of our work is, that different types of NDT measurements (eddy current and magnetic hysteresis) were performed on the same series of samples. The results of different measurements have been compared with each other and conclusions have been drawn for applicability of these techniques.

\section{Specimens}

\subsection{Sample preparation}

The injectors, which are studied in this work, are parts of the so called Common Rail System of diesel engines [15]. Their base material is a DSCM415H-AS carbon steel, the chemical composition is given in Table 1.

Fabrication of the injectors is a rather complicated industrial process, and to go to the details is not the purpose of this paper. The base material is first forged and then annealed and trained. Annealing means a low pressure cementation [16]. During cementation carbon content of the surface of the low carbon 
Table 1

The chemical composition of the base material given in weight $\%$

\begin{tabular}{cccccccccccc}
\hline $\mathrm{C}$ & $\mathrm{Si}$ & $\mathrm{Mn}$ & $\mathrm{P}$ & $\mathrm{S}$ & $\mathrm{Cr}$ & $\mathrm{Ni}$ & $\mathrm{Cu}$ & $\mathrm{Mo}$ & $\mathrm{Bi}$ & $\mathrm{Ti}$ & $\mathrm{Fe}$ \\
\hline $0.12-$ & $0.15-$ & $0.55-$ & $0.030 \max$ & $0.003 \max$ & $0.85-$ & $0.25 \mathrm{max}$ & $0.30 \mathrm{max}$ & $0.15-$ & $0.015-$ & $0.004-$ & $\mathrm{Rest}$ \\
0.18 & 0.35 & 0.90 & & & 1.25 & & & 0.35 & 0.025 & 0.008 & \\
\hline
\end{tabular}

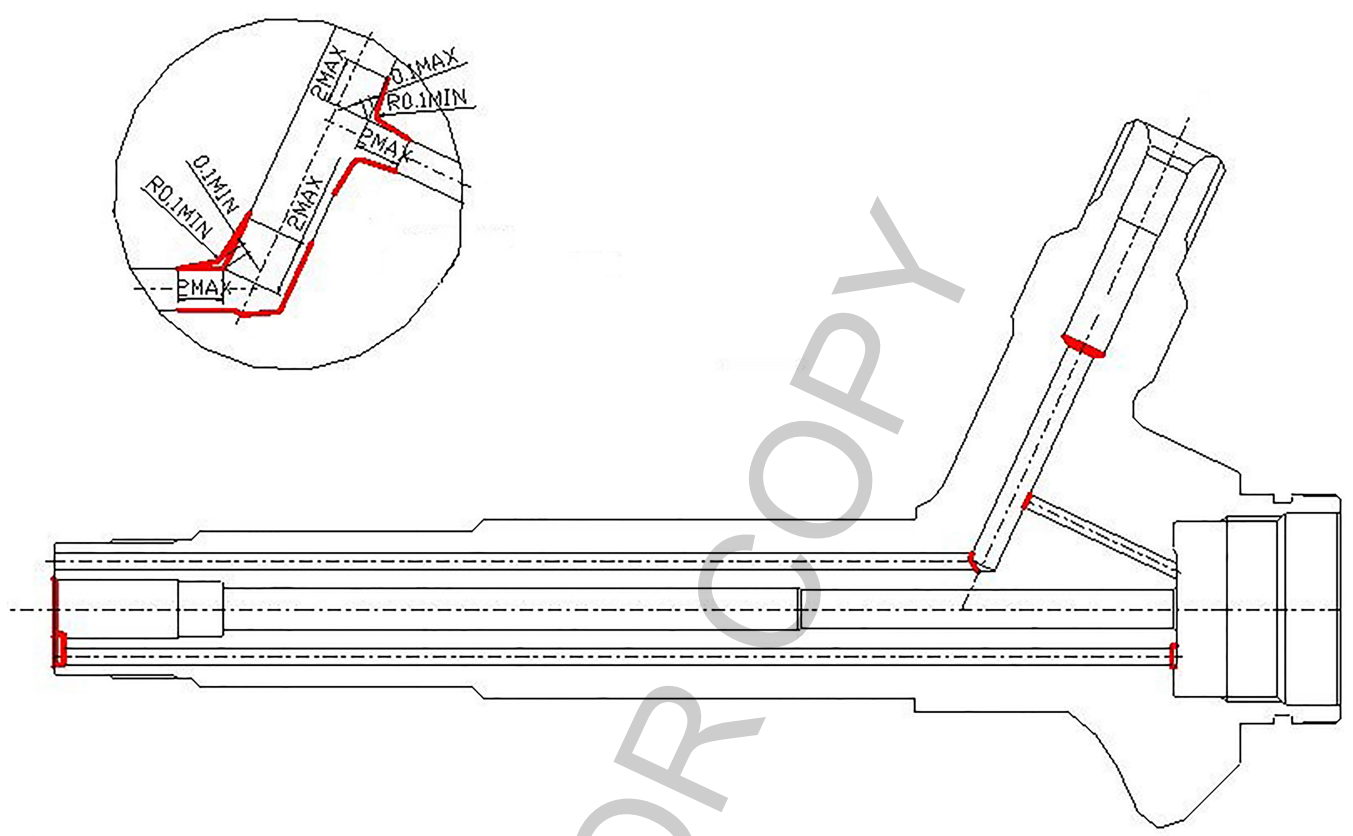

Fig. 1. Schematic cross section of the injector. The total length is $180 \mathrm{~mm}$.

steel is increased. The thickness and the carbon content of this hardened layer depends on quality of the steel and on conditions of the cementation process. The thickness of the trained surface layer should be $0.8 \pm 0.2 \mathrm{~mm}$. This is the important parameter that should be determined as precisely as possible. The inspection of the surface layer is performed by the TCD method, which is practically measurement and evaluation of its hardness profile.

Our measurements were performed on pieces of injectors, which already underwent this training process. The schematic cross section of the injector is given in Fig. 1.

The purpose of our work is to determine thickness of the trained surface layer by electromagnetic nondestructive methods. In the fabrication technology this thickness is modified by conditions of the heat treatment. However, for our tests of the methods we started with ten identical injectors with the (assumed) identical thicknesses of their hardened layers. In order to ensure the same geometry of the ten samples a $20 \times 19.5 \times 11 \mathrm{~mm}$ size piece was cut from each injector as shown in Fig. 2 (A small rectangular part is missing from the samples, as can be seen in Fig. 2.). The samples were numbered from 1 to 10 , and at the beginning they were considered to be totally equal to each other. Then, to simulate influence of different annealing conditions (and thus different final thicknesses of the hardened surface layers), various thicknesses, $\Delta t$, were mechanically removed from surfaces of the ten samples by a grinding machine. Sample 10 is the reference, virgin sample. From other samples the following $\Delta t$ thicknesses were removed (values are in $\mathrm{mm}$ ): $0.099,0.198,0.255,0.385,0.470,0.580,0.703,0.773$ and 0.906 , respectively. 

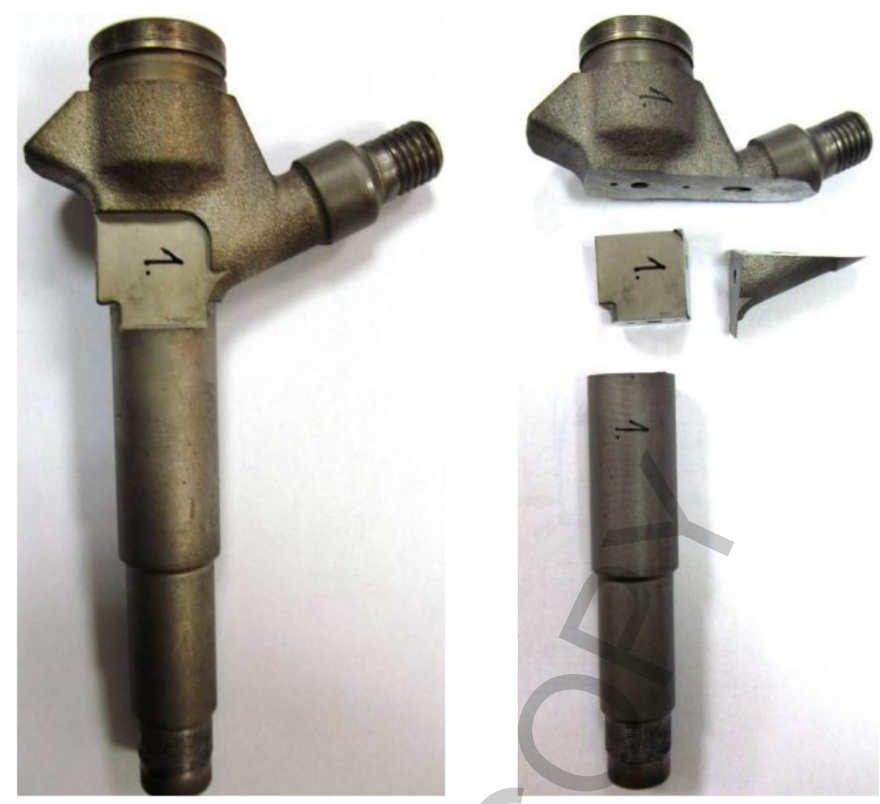

Fig. 2. The injector before (left) and after (right) the cutting of the test sample No.1.

\subsection{TCD (Total Case Depth) technique}

As the first step, a cutting is made at the actual cross section of the heat annealed sample by a precision cutter. Then this sample is prepared for measurement, i.e. it is moulded into a plastic disk, then it is polished to obtain the suitable quality surface for hardness measurement. Polishing is made in five steps by emery wheel, using different granularity water based abrader. Finally the sample is polished ready by using a tucker and by water based tincture conatining $3 \mu \mathrm{m}$ size diamomd grains.

As the second step, a hardness measurement profile is performed. A Micro-Vickers hardness machine is used for this purpose. The applied force is $3 \mathrm{~N}$. Measured points are taken from $0.1 \mathrm{~mm}$ distance from the surface in certain steps. A diagram, as shown in Fig. 3, is produced such a way, where the measured hardness value $(H V)$ are given as a function of the distance. The last point is $2.1 \mathrm{~mm}$ far from the surface. At the moment the total plotting and evaluation is made by hand. As it is illustrated in Fig. 3, a curve is fitted to the measured points. The difference between the maximal and minimal hardness is determined, and at one third of the difference a line is drawn. At the point, where this line crosses the curve a tangent is plotted, and the cross section of this tangent line and minimal hardness line determines the thickness of the trained layer. The whole process is rather time consuming and the error is significant.

\section{Eddy current testing}

\subsection{EddyMax equipment}

The conventional eddyMax Spectra Rack305 equipment (produced by Test Maschinen Technik company, Germany) was used for the investigations. It was possible to perform multi-channel eddy current measurements by using the device's factory default ScanMax software. This application is flexible; there is a wide choice of settings, which ensures parallel investigation of several samples in the frequency 


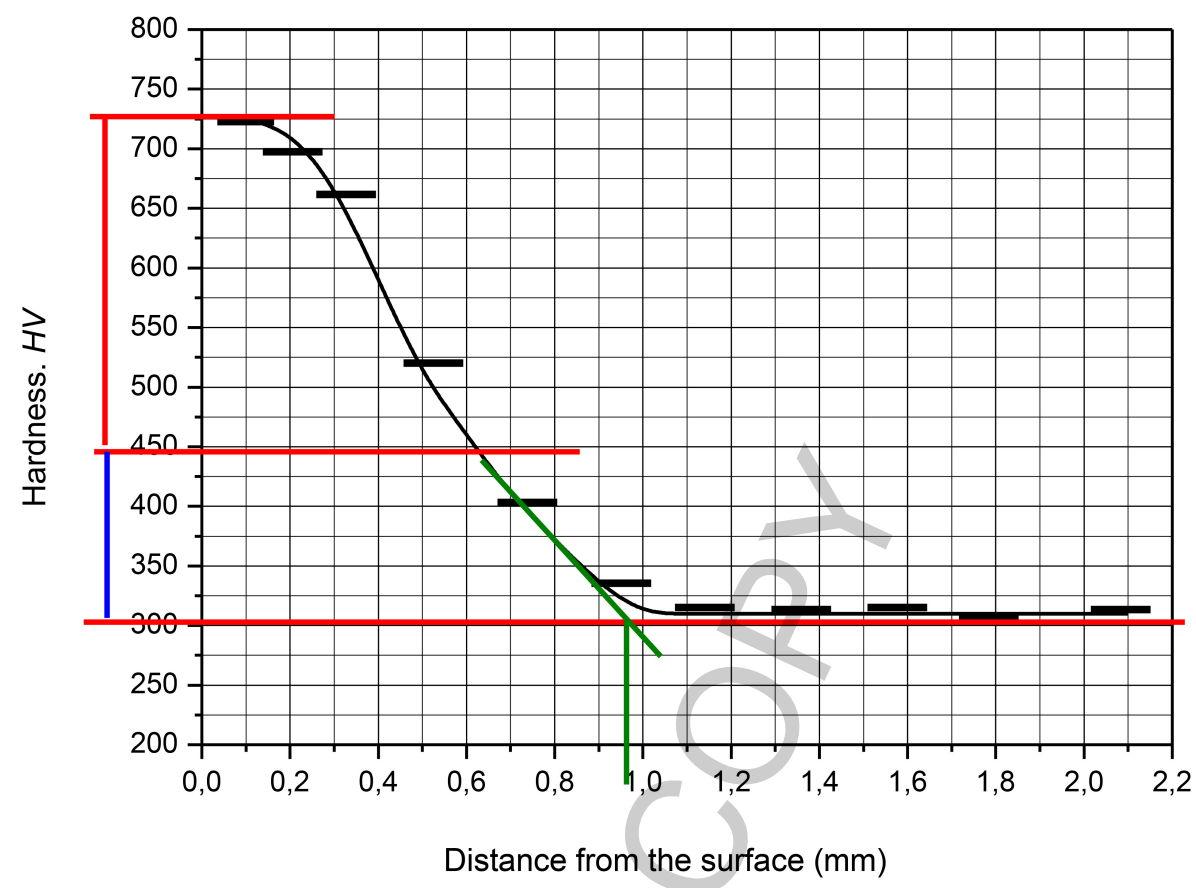

Fig. 3. Illustration TCD (Total Case Depth) technique.

range of $10 \mathrm{~Hz}-2.5 \mathrm{MHz}$, with the $-48 \mathrm{~dB}-126 \mathrm{~dB}$ gain. The device consists of an exciting coil, which generates alternating electromagnetic field in the sample and of a measuring (pick-up) coil. The two coils are built together into a single measuring head.

The measurements were performed in two frequency ranges, to get as much data as possible. The low frequency measurements were done with $100 \mathrm{~Hz}, 133 \mathrm{~Hz}, 160 \mathrm{~Hz}$ and $200 \mathrm{~Hz}$ values, while the high frequency measurements were done with $11.1 \mathrm{kHz}, 50 \mathrm{kHz}, 100 \mathrm{kHz}$ and $200 \mathrm{kHz}$ values. Both the amplitudes and the phase angles were evaluated. The results of the measurements are shown in Fig. 4 for all the frequencies. The amplitudes can be seen in Fig. 4(a), while the phase angles are shown in Fig. 4(b).

Summarizing the results of these measurements, no correlation was found between the eddy current signal and thickness of the trained layer regardless on the applied frequency range, see Fig. 4. The eddyMax equipment and the ScanMax software, which belongs to eddyMax are not suitable to determine thickness of the trained surface layer in our case. The reason probably is, that the commercially available standard ECT devices (such as eddyMax) and their probes are intended to be used mainly for detection of certain material defects (flaws, local inhomogeneities, etc.), i.e. for sensing the microscopic deviations in an otherwise more-or-less homogenous environment.

There are also commercially available devices for measuring insulation layer thickness (i.e. the probe lift-off) over a conducting surface, like painted layer inspectors. For this purpose the traditional inductive coil based method (e.g. [17]) is excellent up to determination of few millimeters of the thickness. In our case, however, neither the typical commercial eddy current probes nor the insulation layer thickness measurement apparatuses can be applied with success. This is, because of the fact that the hardened cemented layer over the steel bulk material cannot be considered as a very different material from the point of view of electric conductivity, or at least the hardened layer is not an electric insulation at all. It is 


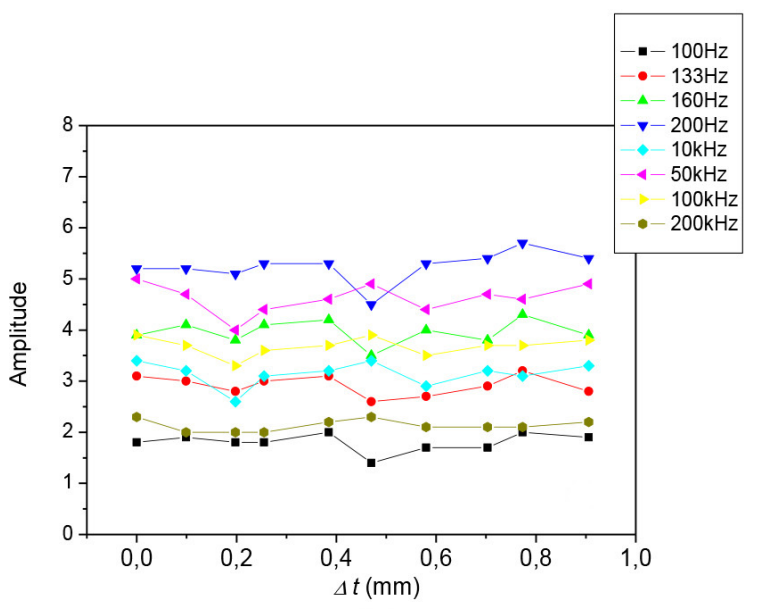

(a)

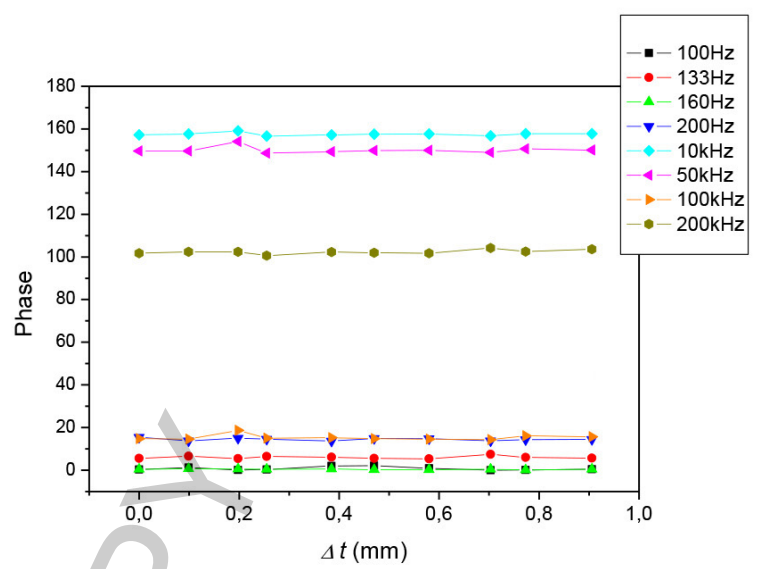

(b)

Fig. 4. The results of the eddyMax measurement in low and in high frequency ranges for the investigated samples. The amplitudes are given in (a), while the phase angles are given in (b).

expected, that not the change in the electrical conductivities between the two sides of the layer wall, but the different magnetic characteristics of the layer has an effect on the distribution of the induced eddy currents.

Another consideration for application of an improved version ECT is that the layer thickness measurement should be based on volumetric (or large area) information rather than on detection of local effects with limited dimensions. The effect of surface roughness and other local microscopic material flaws should be excluded or averaged/filtered out from the probe response, since they are not relevant. These considerations led us to the development of another eddy current testing arrangement.

\subsection{Development of an ECT experimental setup}

A newly developed experimental setup (Setup \#1) for this purpose, and its enhancement (Setup \#2) are described in the following and their obtained results are shown.

\subsubsection{Setup \#1}

In order to get the probe response from a larger volume of the tested material, we increased dimensions of the impedance coil, increasing thereby the excited/interacting volume of the material. We prepared the largest coil that could be used for the samples. Impedance coil type measurement was performed with the single coil without ferromagnetic core. The sketch of the measurement system Setup \#1 is shown in Fig. 5(a). Coil specification: outer diameter $7 \mathrm{~mm}$; inner diameter $1 \mathrm{~mm}$; length $2 \mathrm{~mm}$; number of turns 65 , lift-off minimum $0.5 \mathrm{~mm}$. The impedance coil is connected directly to a FLUKE PM6306 Programmable Automatic LRC Meter. The impedance coil was put onto the tested surface at a fixed position as far as possible from the sample edges. Each of the samples was first demagnetized and then studied at five different frequencies: 50, 60, 100, 300 and $1000 \mathrm{~Hz}$. The coil impedances were measured by the LRC meter.

The real part (i.e. resistance) of the impedance can be seen in Fig. 6. The trend lines coincide with our expectation and also justify applicability of the selected frequencies. Although differences between the tested samples can be distinguished, the signal is weak and the noise level is rather high. 


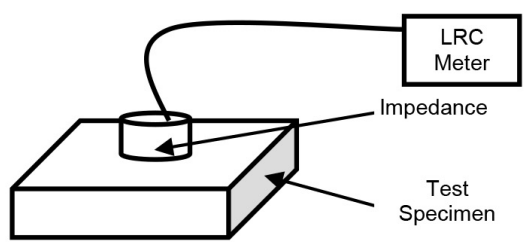

a)

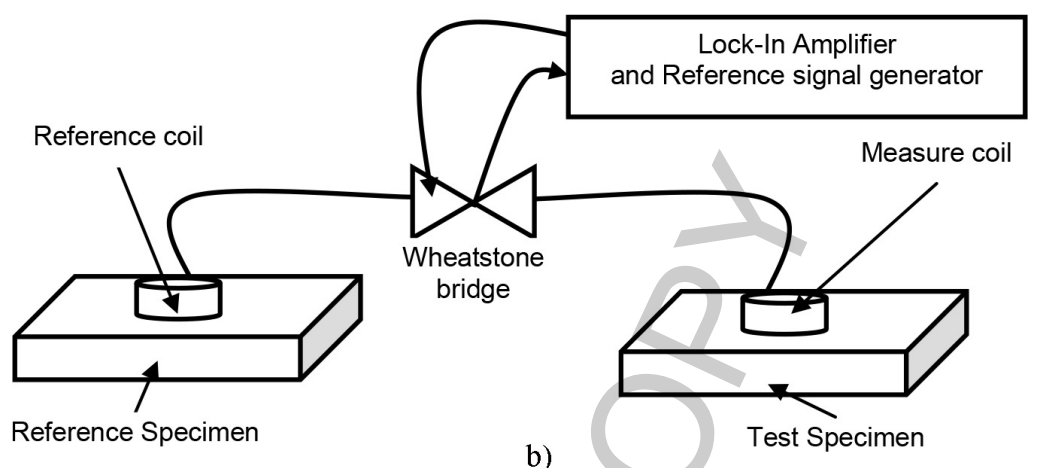

Fig. 5. The sketch of the measurement system Setup \#1 (a) and Setup \#2 (b).

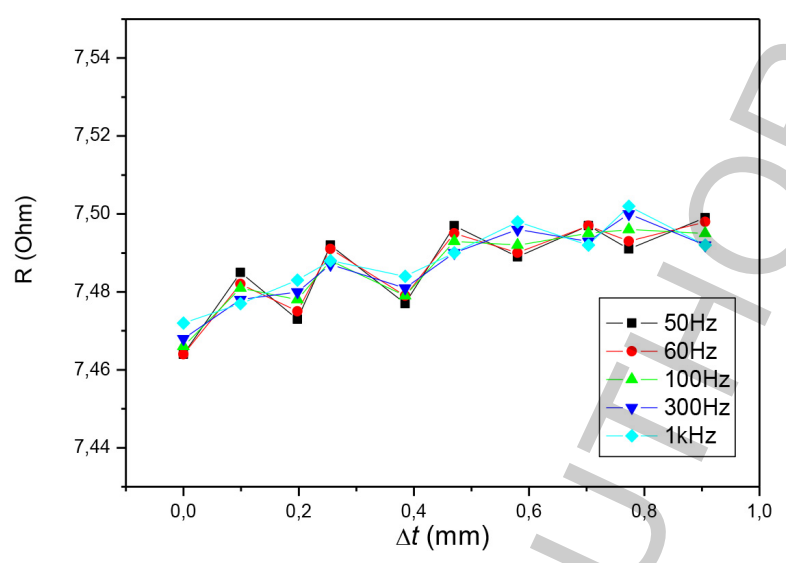

Fig. 6. The real component of the impedance (Setup \#1) as a function of the removed thickness.

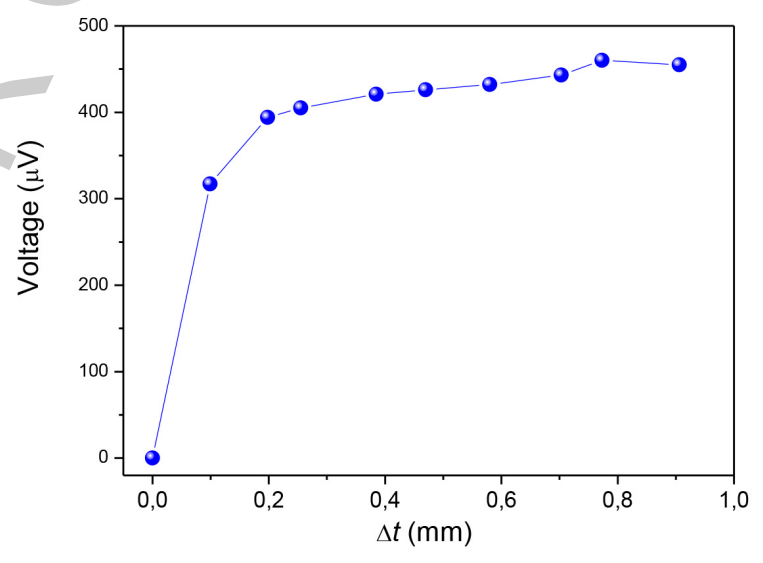

Fig. 7. The output voltage (Setup \#2) as a function of the removed thickness.

\subsubsection{Setup \#2}

In order to improve resolution of the impedance measurement we developed the second experimental setup. It is based on enhancement of the impedance changes. This setup compares the actually tested specimen with a reference one. The Setup \#2 comprises two identical impedance coils (the same as that in Setup \#1), which are connected in a Wheatstone bridge. The well balanced Wheatstone bridge results in a response (i.e. voltage signal) only when the samples have different interaction with the two otherwise identical impedance coils. The sketch of the measurement system Setup \#2 is shown in Fig. 5(b). The coils are placed onto the specimens surfaces at fixed positions as far as possible from the samples edges. The bridge is driven by, and the bridge response is processed by a Lock-In Amplifier with a built-in reference signal generator. Type is Standford Research Systems SR830 DSP Lock-In Amplifier. In this 
case only one frequency value $(150 \mathrm{~Hz})$ was used, which ensured a high enough penetration depth. The impedance coil parameters at $150 \mathrm{~Hz}$ frequency: resistance $0.02 \mathrm{Ohm}$; inductance $32 \mu \mathrm{H}$.

The processed experimental results of the Setup \#2 can be seen in Fig. 7. It can be concluded, that the second experimental setup was found suitable for distinguishing between samples with different layer thickness with a good signal/noise ratio. The results are coherent and they correspond to our expectations.

\section{Magnetic Adaptive Testing}

This method is a special type of magnetic hysteresis technique, which is based on systematic measurement and evaluation of magnetic minor hysteresis loops $[18,19]$. Magnetic Adaptive Testing (MAT) investigates a complex set of minor hysteresis loops (increasing the magnetizing amplitude from a minimum up to a chosen maximum by regular steps) for each sample of the measured series. It follows from the theory of Preisach model of hysteresis [20], that such a set of experimental data contains complex information on hysteresis of the measured material and through that also on its microstructure quality.

A specially designed Permeameter with a soft yoke was applied for magnetization of the samples and measurement of families of minor loops of the sample-yoke magnetic circuit. Size of the yoke was chosen to fit geometry of the samples: it was a C-shaped laminated $\mathrm{Fe}-\mathrm{Si}$ transformer core with crosssection $S=10 \times 5 \mathrm{~mm}^{2}$, the total outside length $18 \mathrm{~mm}$, and the total outside height of the bow $22 \mathrm{~mm}$. The magnetizing coil was wound on the bow of the yoke, with $N=200$ turns and the pick-up coil was wound on one of the yoke legs with $n=75$ turns.

The magnetizing coil gets a triangular waveform current with step-wise increasing amplitudes and with a fixed slope in all the triangles. This produces a triangular time-variation of the applied field in the magnetizing circuit and a signal is induced in the pick-up coil. As long as the field sweeps linearly with time, the voltage signal in the pick-up coil is proportional to the differential permeability of the magnetic circuit. The Permeameter works under full control of a PC computer, which registers data-files for each measured family of the minor "permeability loops".

The experimental raw data are then processed by an evaluation program, which divides the originally continuous signal of each measured sample into a family of individual permeability half-loops. The program filters experimental noise and interpolates the experimental data into a regular square grid of elements, $\mu_{i j} \equiv \mu\left(h_{a i}, h_{b j}\right)$, of a $\mu$-matrix with a pre-selected field-step. The coordinates $h_{a i}, h_{b j}$ of the elements represent the actual magnetic field value, $h_{a i}$, on the actual minor loop with the amplitude $h_{b j}$. Each $\mu_{i j}$-element represents one "MAT-descriptor" of the investigated material structure in question.

The matrices are processed by another evaluation program, which divides values of their elements by corresponding element values of a reference matrix (which was measured on a reference sample, here on the sample with $\Delta t=0$ ), and arranges each set of the mutually corresponding elements $\mu_{i j}$ of all the evaluated $\mu$-matrices into a $\mu_{i j}(\Delta t)$-degradation function. For details of the whole MAT procedure see [19].

The degradation functions can be constructed either directly from the signal data ( $\mu$-degradation functions), or alternatively from the first derivative of the data with respect to the magnetizing field ( $\mu^{\prime}$-degradation functions), or from any other derivate or integral of the measured signal. The information content is never increased by differentiation or integration of the signal data, however, some features can become more distinctive or more convenient. In our experiments we found the $\mu$ '-degradation functions to give the best correlation with $\Delta t$. The most sensitive and at the same time most reliable $\mu$ '-degradation function was chosen by analysis of the whole data pool, obtained by the measurements. This correlation is shown in Fig. 7. 


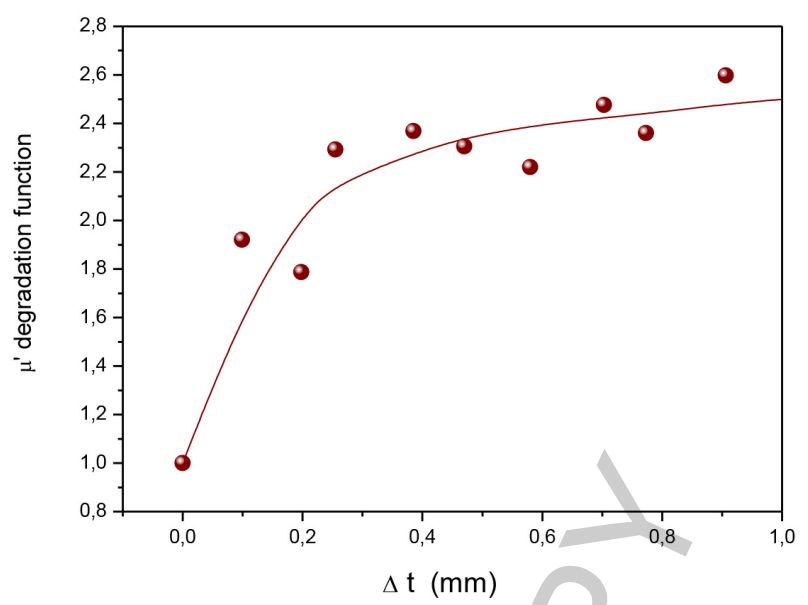

Fig. 8. The optimally chosen MAT descriptor ( $\mu$ ' degradation function) as a function of the removed $\Delta t$ thickness.

\section{Discussion}

Three different ways of eddy current testing and one magnetic hysteresis method were applied for inspection of thickness of the trained surface layer of the diesel engine injectors. The commercial eddyMax equipment was found not suitable for this problem. The penetration depth of the electromagnetic field for this type of steel material (conductivity $1 \mathrm{MS} / \mathrm{m}$, relative permeability 100) is $5 \mathrm{~mm}$ if $100 \mathrm{~Hz}$ excitation is used. It means that the bad sensitivity of the classical EC method cannot be explained in term of penetration depth compared to the hardened layer thickness.

The improved versions of ECT devices and also the Magnetic Adaptive Testing method resulted in reasonable monotonic correlations between the electromagnetically measured quantities and thickness of the trained layer. It feels satisfactory, that all the three "successful" methods give correlations of the same type. Evidently, a properly chosen electromagnetic method can be certainly used for this type of inspection.

The eddy current Setup \#1 revealed better sensitivity, than the commercial eddyMax device. The better sensitivity is attributed to the larger volume of excitation and the high sensitivity of the applied LRC Meter. Howevere it is still on the limit of a reasonable detection. Because of this, eddy current Steup \#1 cannot be suggested for future practical application, because modification of the measured signal between the two extreme $\Delta t$ values is less than $1 \%$ and the scatter of points is high.

In contrast, the eddy current Setup \#2 revealed a significant increase of the output signal between the two extreme $\Delta t$ values and the error of measurement is low. This improved sensitivity is attributed to the differencial mode of signal processing. This setup is a promising candidate for practical applications. Among main advantages of this method is simplicity and low cost of the measuring equipment and speed and reliability of the measurement. Requirement of the reference sample during the measurement can be - perhaps - considered as its drawback.

As for the MAT method, its sensitivity is good, but scatter of points is rather large. The way of measurement is a little bit more complicated than that of the ECT methods: a magnetizing yoke must be attached to the sample surface (in order to magnetize the sample enough) and several minor loops should be measured and evaluated in order to find the optimum MAT parameters for the case. However, after this "teaching procedure", a single measurement is enough for determination of the trained layer thickness of unknown samples of the same kind, if parameters of the measurement are kept the same as for 
the reference sample. Generally, however, thickness measurement by MAT of a magnetically harder thin layer on a magnetically softer bulk is evidently much less convenient than the opposite case, which is discussed in [21].

\section{Conclusions}

Both the properly modified eddy current technique and the method of Magnetic Adaptive Testing were shown as principally suitable for nondestructive inspection of thickness of the hardened surface layer of the diesel engines steel injectors. A monotonic correlation with enough sensitivity and acceptable signal/noise ratio between the electromagnetic parameters and thickness of the hardened layer were found. Based on the presented calibration curves, determination of the thickness of hardened layers can be done on unknown samples of the same kind.

Another message of the present work is that the type of correlation between the hardened layer thickness and the nondestructively measured electromagnetic characteristics is very similar to each other regardless on type of the applied method.

For future practical applications the modified ECT Setup \#2 seems to be the most promising.

\section{Acknowledgments}

The work was supported by the Hungarian Scientific Research Fund (project K 111662) and by the Researcher Exchange Program between the Czech Academy of Sciences and Hungarian Academy of Sciences. One of the co-authors (I.T.) appreciates support of the project No.14-36566G of the Czech Science Foundation.

\section{References}

[1] D.C. Jiles, Magnetic methods in nondestructive testing, D.C. Jiles, Encyclopedia of Materials Science and Technology, p. 6021. Ed. K.H.J. Buschow et al., Elsevier Press, Oxford, September 2001.

[2] C.C.H. Lo, A review of the Barkhausen effect and its applications to nondestructive evaluation, Feature Article in Materials Evaluation, July issue 2004, 741-748.

[3] G. Bach, K. Goebbels and W.A. Theiner, Characterization of Hardening Depth by Barkhausen Noise Measurement, Materials Evaluation 46 (1988), 1576-1580.

[4] S. Vaidyanathan, V. Moorthy, T. Jakakumer and B. Raj, Evaluation of induction hardened case, depth through microstructural characterization using magnetic Barkhausen emission technique, Materials Science and Technology 16 (2000), 202-208.

[5] V. Moorthy, B.A. Shaw and K. Brimble, Testing of case depth in case carburized gear steels using magnetic Barkhausen emission technique, Materials Evaluation 62 (2004), 523-527.

[6] M. Johnson, C. Lo, S. Hentscher and E. Kinser, Analysis of Conductivity and Permeability Profiles in Hardened Steel., Electromagnetic Nondestructive Evaluation, Volume IX. IOS Press, 2005.

[7] C.C.H. Lo, E.R. Kinser, Y. Melikhov and D.C. Jiles, Magnetic nondestructive characterization of case depth in surfacehardened steel components, Review of Quantitative Nondestructive Evaluation, Vol. 25 ed. By D.-O. Thompson and D.E. Chimenti, 2006 American Institute of Physics, pp. 1253-1260.

[8] V. Augutis, R. Amūnas Ramanauskas, A. Ciuplys, J. Vilys and V. Ciuplys, Determination of Metal Surface Hardened Layer Depth Using Magnetic Barkhausen Noise, Materials Science (Medžiagotyra) 12 (2006), 84-897.

[9] C. Zhang, N. Bowler and C.C.H. Lo, Magnetic characterization of surface-hardened steel, Journal of Magnetism and Magnetic Materials 321 (2009), 3878-3887.

[10] H. Sun, J. Bowler, N. Bowler and M. Johnson, Eddy current Measurements on Case Hardened Steel, Review of Progress in Quantitative Nondestructive Evaluation 21 (2001), 1561-1568. 
[11] K.V. Rajkumar, B.P.C. Rao, B. Sasi, A. Kumar, T. Jayakumar, B. Raj and K.K. Ray, Characterization of aging behaviour in M250 grade maraging steel using eddy current non-destructive methodology, Mater. Sci. Eng. A 464 (2007), 233-240.

[12] S. Konoplyuk, T. Abe, T. Uchimoto, T. Takagi and M. Kurosawa, Characterization of ductile cast iron by eddy current method, J. NDT\&E Int 38 (2005), 623-626.

[13] T. Uchimoto, T. Takagi, S. Konoplyuk, T. Abe, H. Huang and M. Kurosawa, Eddy current evaluation of cast irons for material characterization, J. Magn. Magn. Mater 258-259 (2003), 493-496.

[14] M. Hossein Nateq, S. Kahrobaee and M. Kashefi, Nondestructive characterization of induction hardened cast iron parts, in: 2nd International Conference on Materials Heat Treatment, International Conference on Materials Heat Treatment (ICMH 2011), Isfahan, Iran, 2011.

[15] http://www.mpdiesel.hu/index.php.

[16] http://www.mtvac.com/MTV_Presentation_full.pdf.

[17] Siemens Testcenter, http://www.khe.siemens.de/IT/.

[18] I. Tomáš, Non-Destructive Magnetic Adaptive Testing of Ferromagnetic Materials, J. Magn. Magn. Mater 268(1-2) (2004), 178-185.

[19] I. Tomáš and G. Vértesy, Magnetic Adaptive Testing, in: Nondestructive Testing Methods and New Applications, M. Omar (Ed.), ISBN: 978-953-51-0108-6, (2012), InTech: http://www.intechopen.com/articles/show/title/magneticadaptive-testing.

[20] I.D. Mayergoyz, The classical Preisach model of hysteresis and reversibility, J. Appl. Phys 69 (1991), 4602-4604.

[21] O. Perevertov, O. Stupakov, I. Tomáš et al., Detection of spring steel surface decarburization by magnetic hysteresis measurements, Ndt \& E International 44 (2011), 490-494. 